\title{
Statistical analysis of locomotives traction motors performance
}

\author{
Oleksander Gorobchenko ${ }^{1}$, Victor Tkachenko ${ }^{1 *}$ \\ ${ }^{1}$ State University of Infrastructure and Technologies, Department of Traction Railway rolling stock, Kyiv, Ukraine
}

\begin{abstract}
In this article the authors quoted results of traction motors speed performance variations statistical analysis and wage wheels bands diameters deviations according to operation data. The authors of this publication offered the method of determining the non-uniformities of current distribution in the power circuit of the locomotive, based on probabilistic approach, and also performed its comparison with classic computation approach. The next statistical performance of the continuous current locomotives traction drive is as follows: mathematical expectation of the motors magnetic flux; mean square deviation of magnetic flux; statistical dispersion and average square deviation of wage wheels bands diameters. With help of the received data it became possible to improve determination of non-uniformities currents deviations on traction motors during loading of the electric locomotives.
\end{abstract}

\section{Introduction}

As operation experience says, efficiency of the locomotives work can be significantly increased provided that we use trailing weight, power of traction electric drives to a fuller extent and improve the traction electric drives control systems [1,2] .

The existing diagrams and control algorithms do not allow to implement potentialities of traction rolling stock concerning using of trailing weight and power of electric drives completely. One of the main reasons is nonuniformity of using the power of the drives.

The main method to improve performance of the locomotives is to develop devices for the locomotive traction control system altogether and for each wage wheels individually [3, 4]. Most of the locomotives, used at the railways of post-Soviet countries, are equipped with classic control systems $[5,6]$, which do not provide control of load reallocation on axes. So determination and elimination of factors, which have an impact on inequality of the current distribution at the power circuits of the locomotives is a currently central issue. When we solve this issue, it will allow us to improve traction output performance of the existing rolling stock.

\section{Problem discussion}

The purpose of this work is to obtain statistical values of traction electric transmission parameters distribution. This will allow to justify the methods of eliminating the non-uniformities of current distribution at the power circuits of the locomotive wage wheels drive in a more qualitative manner.

We found that the crucial influence on current distribution at the power circuits is caused by such factors as velocity performance of traction electric motors, surfaces wheels rolling wage wheels diameters, resistance of the electric motors electric coils [7-9].

There is a small probability that in one locomotive at the parallel paths we find the motors with extreme deviations of velocity performance [10]. In this case we will have disadvantageous combination of them with wage wheels.

We need to use the data which are closer to reality when we forecast tendency to non-uniform current distribution of the locomotives. According to results of calculations we can conclude that using of special devices to flatten the currents for particular brands of the locomotives is rational.

\section{Analysis of traction drives velocity performance variations according to operation data}

To analyze velocity performance of traction motors we made a data retrieval from tests reports of electric locomotive continuous current traction electric motors with voltage in the contact system $3000 \mathrm{~V}$. We analyzed 428 motors. We analyzed performances of the motors during clockwise and anticlockwise rotation. The results of statistical analysis are on Fig. 1 and 2. We measure frequency of the motors rotation during rated one hour's duration current, which is $380 \mathrm{~A}$.

Average rotation frequency

$$
\bar{n}_{m}=\frac{1}{z} \sum_{i=1}^{k} N_{i} z_{i}
$$

where $z_{i}$ is a number of motors in i-type rotation frequency interval;

$z$ - general number of motors

$k$ - number of intervals; 
$\boldsymbol{N}_{\boldsymbol{i}}$ - number of motors, which are classified to interval by test results.

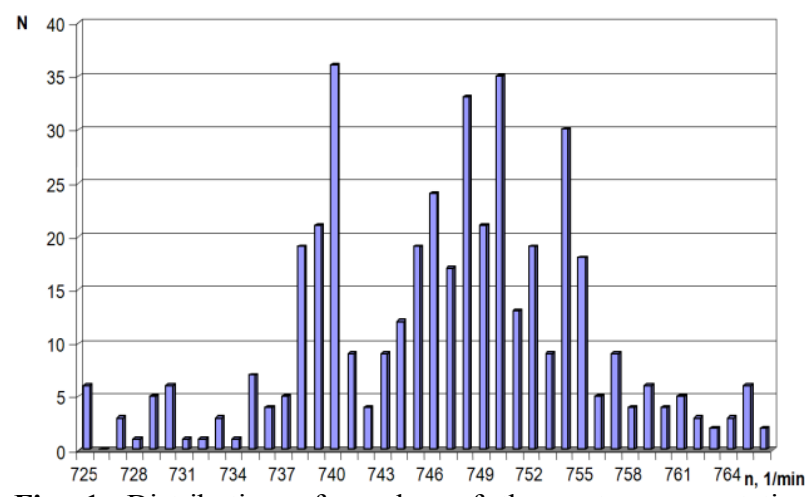

Fig. 1. Distribution of number of the motors on rotation frequencies during clockwise rotation $-\bar{n}_{m r}$.

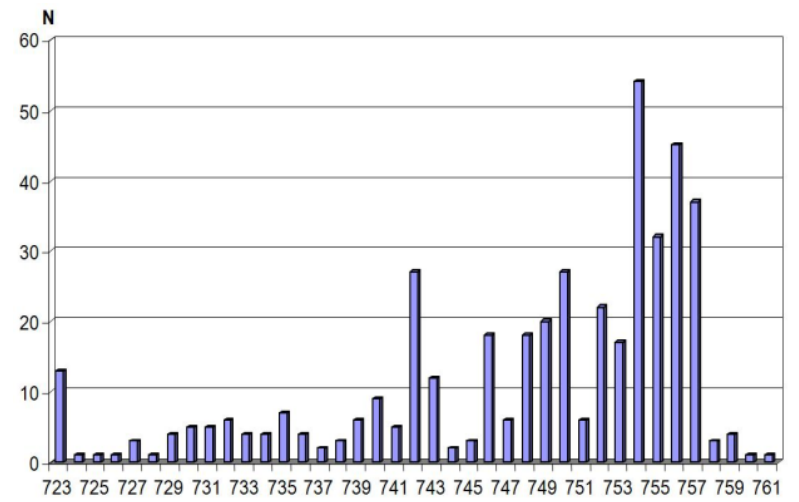

$\mathrm{n}, 1 / \mathrm{min}$

Fig. 2. Distribution of number of the motors on rotation frequencies during anticlockwise rotation $-\bar{n}_{m l}$.

Analysis results: $\bar{n}_{m r}=746,8 \mathrm{rpm} ; \bar{n}_{m l}=748 \mathrm{rpm}$; $\bar{n}_{m}=747,45 \mathrm{rpm}$.

Therefore, average motors rotation frequency in operation is exaggerated comparing to rated one (735 rpm).

Rotations frequency dispersion:

$$
D_{n}^{*}=\frac{1}{z} \sum_{i=1}^{k}\left(N_{i}-\bar{n}_{c p}\right)^{2} z .
$$

When we put in the data on rotation frequencies, we will obtain for clockwise rotation of traction electric motors $\mathrm{D}^{*}{ }_{\mathrm{nmr}}=67,15$, for anticlockwise rotation of traction electric motors $-\mathrm{D}^{*}{ }_{\mathrm{nml}}=81,15$.

Statistical average square deviation of rotation frequencies by formula

$$
\sigma_{\mathrm{n}}^{*}=\sqrt{D_{n}^{*}}
$$

During calculation we obtain (for clockwise and left side anticlockwise rotation):

$\sigma_{\mathrm{nmr}}^{*}=8,19$;

$\sigma_{\mathrm{nml}}^{*}=9$.

Due to the fact that difference of velocity performance of only one locomotive influences nonuniformity of current distribution, we processed statistical data concerning ultimate difference of rotation frequencies within one locomotive. As a result we obtained the following set of data, which are shown as histogram on Fig. 3 and 4.

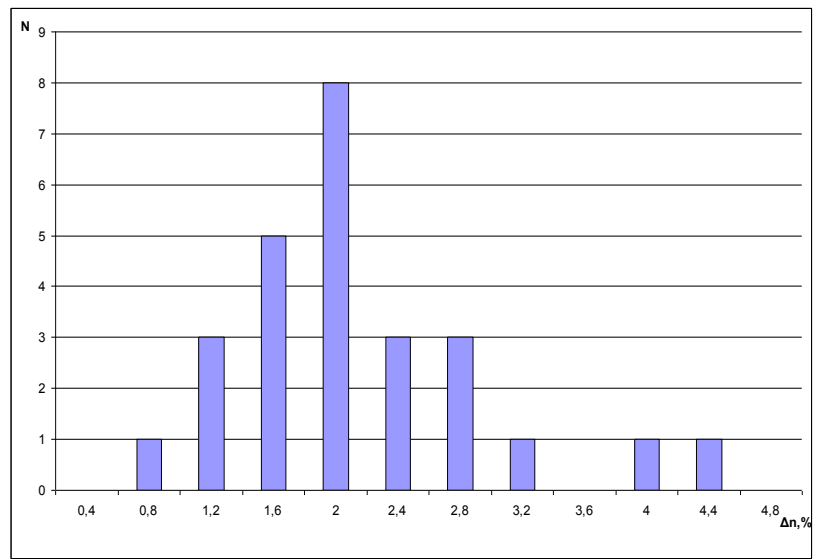

Fig. 3. Distribution of deviations in difference of the velocity performances within one locomotive during clockwise rotation of the traction electric motors.

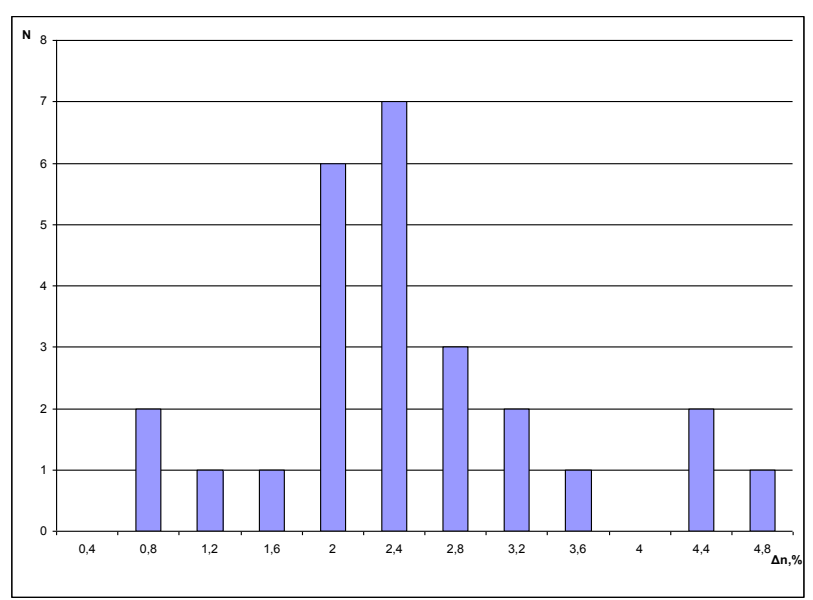

Fig. 4. Distribution of deviations in difference of the velocity performances within one locomotive during anticlockwise rotation of the traction electric motors.

On the ground of the statistical data processing we obtained the following results:

- average deviation of performances within one locomotive:

- during clockwise rotation of the traction electric motors $-\Delta \mathrm{n}=2,13 \%$;

- during anticlockwise rotation of the traction electric motors $-\Delta \mathrm{n}=2,5 \%$;

- statistical dispersion: $\mathrm{D}_{\mathrm{n} \% \mathrm{r}}^{*}=0,664, \mathrm{D}_{\mathrm{n} \% \mathrm{l}}^{*}=0,955$;

- average square deviation $\sigma^{*}{ }_{\mathrm{n} \% \mathrm{r}}=0,815, \sigma^{*}{ }_{\mathrm{n} \% \mathrm{l}}=0,977$.

\section{Statistical analysis of locomotives rolling wheel surfaces diameters deviations according to operation data}

In order to consider impact of locomotives rolling wheel surfaces diameters spread on non-uniformity of the current distribution between the traction electric motors we conducted research of the continuous current electric locomotive fleet, which are operated on Ukrzaliznytsia Ukrainian railway, namely 40 locomotives of ВЛ8 
series. As a result we obtained statistical data, shown on Fig.5.

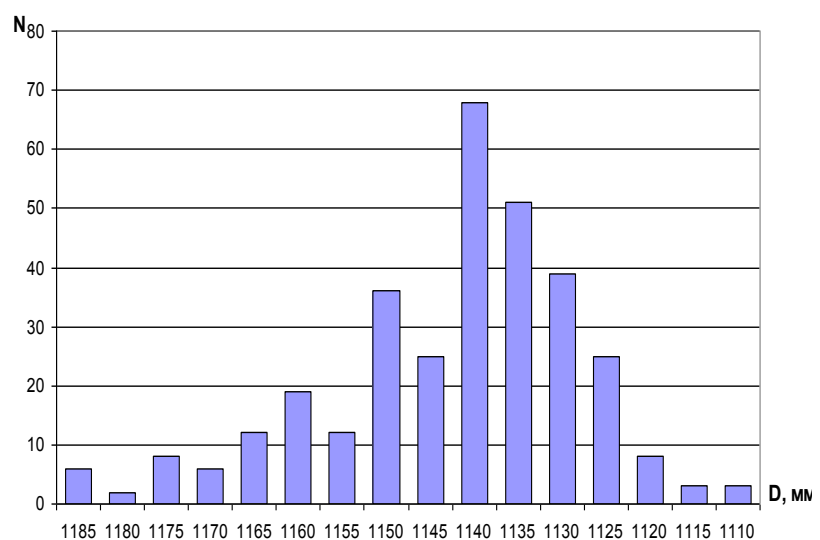

Fig. 5. Distribution of wheel pairs of locomotives on the diameters of rolling surfaces of wheels.

Average value of bands diameters $D$ is calculated using the formula

$$
\bar{D}=\frac{1}{n} \sum_{i=1}^{k} D_{i} n_{i}
$$

where $n$ is general number of wheels;

$k$ - number of intervals;

$D_{i}$ - average interval value of wheels diameters;

$n_{i}$ - number of wage wheels, classified to interval according to results of measurements.

Dispersion of wheels diameters $D_{D}$ are found using the formula:

$$
D_{D}^{*}=\frac{1}{n} \sum_{i=1}^{k}\left(D_{i}-\bar{D}\right)^{2} n_{i}
$$

Statistical average square deviation of wheels diameters is found using the formula $\sigma_{D}^{*}=\sqrt{D_{D}^{*}}$.

We obtained the following performances:

- average value of wheels diameters: $\bar{D}=1142$ $\mathrm{mm}$;

- dispersion of wheels diameters: $D_{D}=214 \mathrm{~mm}$;

- statistical average square deviation of wheels diameters $-\sigma_{D}=14,63$.

The crucial thing is that spread of wheels diameters within one section of the locomotive has an impact on nonuniformity of current distribution. We presented the obtained results on Fig. 6.

On the ground of processing of the selected material we obtained the following results:

- average value of ultimate difference of diameters $\Delta D=13,9 \mathrm{~mm}$;

- statistical dispersion $D_{D}=14,95$;

- statistical average square deviation $\sigma_{D}=3,87$.

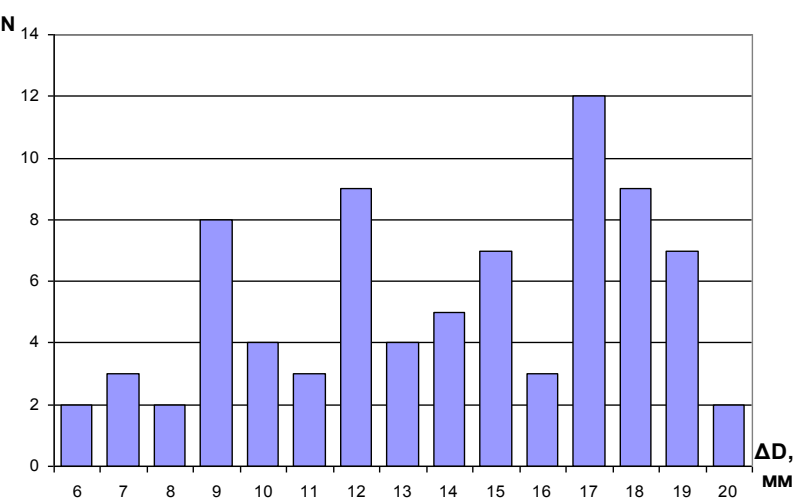

Fig. 6. Distribution of locomotives on the difference in the diameter of the rolling surfaces of the wheels within the limits of one section of the electric locomotive.

\section{Correlation of the current distribution non-uniformities, calculated on limiting and probabilistic laws}

Magnetic flux of i-type drive $\Phi_{i}$ is a normal random variable, [11]

$$
f_{1}\left(\Phi_{i}\right)=\frac{1}{\sigma_{1} \sqrt{2 \pi}} e^{\frac{-\left(x-m_{1}\right)}{2 \sigma_{1}^{2}}},
$$

where $m_{l}$ is a moral expectation $\Phi_{i}$;

$\sigma_{1}$ - average square deviation $\Phi_{i}$;

Also there is the other normal random variable wheels rolling surfaces diameters $D_{b}$

$$
f_{1}\left(D_{b}\right)=\frac{1}{\sigma_{2} \sqrt{2 \pi}} e^{\frac{-\left(y-m_{2}\right)}{2 \sigma_{2}^{2}}},
$$

where $m_{2}$ is a moral expectation $D_{b}$;

$\sigma_{2}$ - average square deviation $D_{b}$.

It is natural to suppose that random variables magnetic flux and bands diameters - are independent. In this case density of random variables system distribution $\Phi_{i}$ and $D_{b}$ looks like $f\left(\Phi_{i}, D_{b}\right)=f_{1}\left(\Phi_{i}\right) f_{2}\left(D_{b}\right)$.

Spheres $S$ (Fig. 7), in which there will be parameters $\Phi_{i}$ and $D_{b}$ with probability $\gamma$, is found by means of calculating the integral

$$
\begin{gathered}
\gamma=\iint_{S} f(x, y) d x d y=\iint_{S} f_{1}\left(\Phi_{i}\right) f_{2}\left(D_{b}\right) d \Phi_{i} d D_{b}= \\
=\int_{a_{1}}^{b_{1}} f_{1}\left(\Phi_{i}\right) d \Phi_{i} \int_{a_{2}}^{b_{2}} f_{2}\left(D_{b}\right) d D_{b},
\end{gathered}
$$

where $a_{1}, b_{1}, a_{2}, b_{2}$ - are limits of the sphere of change of parameter $\Phi_{i}$ and $D_{b}$.

It is natural to choose the intervals, situated symmetrically towards moral expectation of the random variable. 


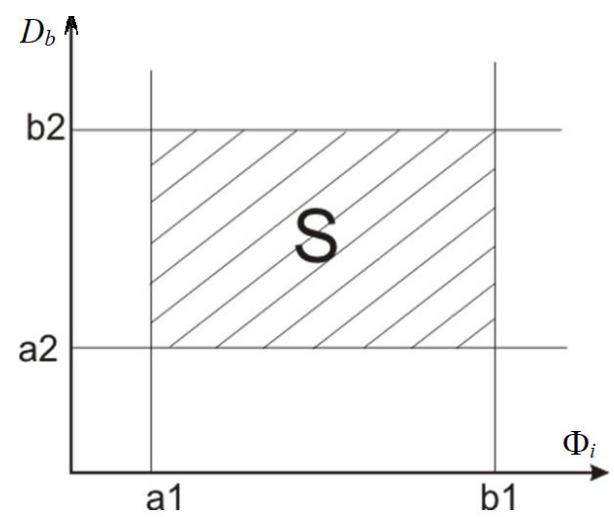

Fig. 7. Sphere of change of parameters $\Phi_{i}$ and $D_{b}$.

As we can see from the Fig. 8 and 9, for the random variable $\Phi_{i}$ we can choose the intervals $[\mathrm{m} 1-\mathrm{c} 1 ; \mathrm{m} 1+\mathrm{c} 1]$, ad for the random variable $D_{b}-$ interval [m2-c2; $\mathrm{m} 2+\mathrm{c} 2]$.

Probabilities of penetration of the random variables $\Phi_{i}$ and $D_{b}$ into the considered intervals are calculated using the following formulas:

$$
\begin{aligned}
& P_{1}=P\left(m_{1}-c_{1}<\Phi_{i}<m_{1}+c_{1}\right)=P\left(\left|\Phi_{i}-m_{1}\right|<c_{1}\right)=2 F\left(\frac{c_{1}}{\sigma_{1}}\right) \\
& P_{2}=P\left(m_{2}-c_{2}<D_{b}<m_{2}+c_{2}\right)=P\left(\left|D_{b}-m_{2}\right|<c_{2}\right)=2 F\left(\frac{c_{2}}{\sigma_{2}}\right)
\end{aligned}
$$

where $F(x)=\frac{1}{2 \pi} \int_{0}^{x} e^{-\frac{t^{2}}{2}} d t$ is a Laplace integral.

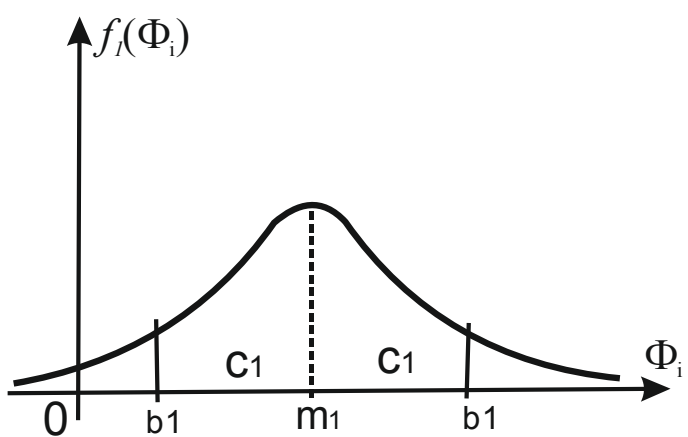

Fig. 8. Curvature of distribution density for the magnetic flux.

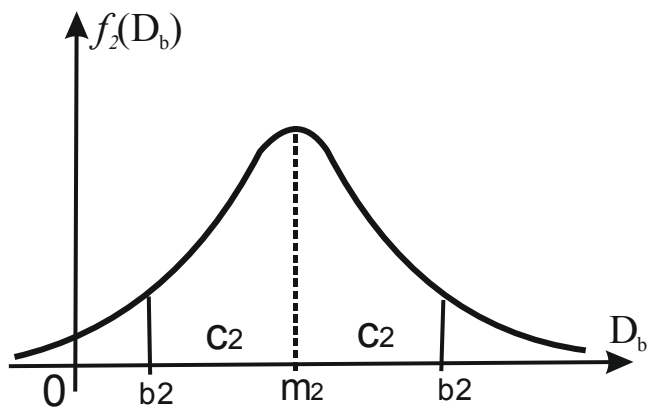

Fig. 9. Curvature of the wheels diameters density.

The function $F(x)$ is a tabulated function. The tables of its values are known from [11].

The variables $c_{1}$ and $c_{2}$ are chosen provided that

$$
P_{1} P_{2}=4 F\left(\frac{c_{1}}{\sigma_{1}}\right) F\left(\frac{c_{1}}{\sigma_{1}}\right) \geq \gamma,
$$

where $\gamma$ is chosen, coming from engineering and operation conditions.

In rated static mode the current of the traction electric motor is determined using the known formula

$$
I=\frac{U-c_{E} n \Phi}{\mathrm{R}},
$$

where $U$ is voltage input, $V$;

$c_{E}$ is a constant by electromotance;

$n$ is a frequency of the motor rotation, rpm;

$\Phi$ - magnetic flux, $\mathrm{Wb}$;

$R$ - equivalent motor resistance, $\Omega$.

Increase of the current is described by the following dependence

$$
\Delta I=\frac{\partial I}{\partial n} \Delta n+\frac{\partial I}{\partial \Phi} \Delta \Phi
$$

where $\frac{\partial I}{\partial n}=-\frac{C_{E} \Phi}{R} ; \quad \frac{\partial I}{\partial \Phi}=-\frac{C_{E} n}{R}$.

Altogether we obtain

$$
\Delta I=-\frac{C_{E}}{R}(\Phi \Delta n+n \Delta \Phi)
$$

For the simple reason that $n=\frac{60 i V}{3,6 \pi D_{b}}$ and $\Delta n=\frac{60 i V}{3,6 \pi D^{2}{ }_{b}} \Delta D_{b}$ we have

$$
\Delta I=-\frac{60 C_{E} i V}{3,6 \pi R D_{b}}\left(\frac{\Phi}{D_{b}} \Delta D-\Delta \Phi\right),
$$

where $i$ is a reduction ratio of thrust actuator;

$$
V \text { - a vehicle unit velocity, } \mathrm{km} / \mathrm{h} \text {. }
$$

\section{Conclusions}

We determined how difference between traction electric motors and diameters of the locomotives wheels impacts on distribution of currents in electric locomotives drive power circuits. We developed the method of calculating parameters of current distribution and took into account allowances on traction electric motors and practical deviations of their performances during operation. The proposed method gives us the opportunity to evaluate non-uniformity of traction electric motor current distribution in broad limits of engineering and operation allowances.

In accordance with statistical data for continuous current electric locomotives we obtained the following variables: moral expectation of the motors magnetic flux $\mathrm{m}_{1}=0,146 \mathrm{~Wb}$, average square deviation of magnetic flux $\sigma_{1}=7 \cdot 10^{-4} \mathrm{~Wb}$, For moral expectation of the bands 
diameters we determined $\mathrm{m}_{2}=1142 \mathrm{~mm}$ and average square deviation of the bands diameters $\sigma_{2}=9,92 \mathrm{~mm}$.

According to statistical analysis probability $\gamma=0,9$ will allow the magnetic flux of the motors to change in the interval $(0,14162-0,15038) \mathrm{Wb}$, and bands diameters - in the interval $(1131,7-1152,4) \mathrm{mm}$. For example, for the electric locomotive ДЕ1 non-uniformity of the current distribution in one hour's mode is 32A. The obtained results tell us that the current distribution calculated on the set probabilistic distributions, gets closer to the one obtained thanks to calculations, performed with using maximum allowance.

The results of the presented analysis tell us about availability and degree of tendency of the traction electric motors to overload within one locomotive. It is necessary to take into consideration during repair of the electric locomotives during establishing wheels and motors unit in the bogie frames. The given results it is necessary to take into consideration during setting of the clutch controlling systems and protection of the wage wheels from boxing. When we implement this proposal it will give us the opportunity to improve the traction properties of the locomotives, reduce the expenditures of power on traction and to enhance reliability of capacity value transmission.

\section{References}

1. V. Shamardina, M. Anishchenko, S. Lemeshko, R. Kanunnikov, Functional efficiency enhancement of diesel-electric locomotive traction system. (Modern Electrical and Energy Systems (MEES), IEEE, 2017).

2. E. Mikhailov, S. Semenov, V. Tkachenko, S. Sapronova, Reduction of Kinematic Resistance To. EDP Sciences. 235 (2018). (doi : 10.1051/matecconf/201823500033).

3. M. Spiryagin, P. Wolfs, F. Szanto, C.Cole, Simplified and advanced modelling of traction control systems of heavy-haul locomotives. Vehicle System Dynamics. 53 (2015).

4. T. Ishrat, G. Ledwich, M. Vilathgamuwa, P. Borghesani, Wheel slip control based on traction force estimaton of electric locomotives. Power Engineering Conference (AUPEC), Australasian Universities (1-6). IEEE (2016).

5. R. Hill, Electric railway traction. I. Electric traction and DC traction motor drives. Power Engineering Journal, 8 (1994).

6. S. Nategl, D. Lindberg, O. Aglen, R. Brammer, A. Boglietti, Review and Trends in Traction Motor Design: Electromagnetic and Cooling System Layouts. XIII International Conference on Electrical Machines (ICEM). IEEE (2018).

7. O. Gorobchenko, O. Fomin, V. Fomin, V. Kovalenko, Study of the influence of electric transmission parameters on the efficiency of freight rolling stock of direct current. Eastern-European Journal of Enterprise Technologies, 91 (2018). doi: 10.15587/17294061.2018.121713
8. O. Gorobchenko, Method of calculating currents in the power circuit of an electric locomotive. Collection of scientific papers of the Donetsk Institute of Railway Transport. 5 (2006).

9. O. Gorobchenko, U. Krivosheia, V. Gatchenko, Development of complex mathematical model of electric locomotive electric locomotive using software package Matlab. Collection of scientific papers of the Donetsk Institute of Railway Transport. 13 (2008).

10. A. Steimel, Electric traction-motive power and energy supply: Basics and practical experience. Oldenbourg Industrieverlag (2008).

11. B. Gnedenko, Theory of probability. Routledge (2017). 